\title{
Efficacy of computational predictions of the functional effect of idiosyncratic pharmacogenetic variants
}

\author{
Hannah McConnell ${ }^{\text {Equal first author, } 1}$, T. Daniel Andrews ${ }^{\text {Corresp., Equal first author, } 1}$, Matt A Field ${ }^{\text {Corresp. } 2,3}$ \\ 1 John Curtin School of Medical Research, Australian National University, Canberra, ACT, Australia \\ 2 Australian Institute of Tropical Health and Medicine, Centre for Tropical Bioinformatics and Molecular Biology, James Cook University, Smithfield, \\ Australia \\ 3 Immunogenomics Lab, Garvan Institute of Medical Research, Darlinghurst, NSW, Australia \\ Corresponding Authors: T. Daniel Andrews, Matt A Field \\ Email address: dan.andrews@anu.edu.au, matt.field@anu.edu.au
}

Background. Pharmacogenetic variation is important to drug responses through diverse and complex mechanisms. Predictions of the functional impact of missense pharmacogenetic variants primarily rely on the degree of sequence conservation between species as a primary discriminator. However, idiosyncratic or off-target drug-variant interactions sometimes involve effects that are peripheral or accessory to the central systems in which a gene functions. Given the importance of sequence conservation to functional prediction tools - these idiosyncratic pharmacogenetic variants may violate the assumptions of predictive software commonly used to infer their effect.

Methods. Here we exhaustively assess the effectiveness of eleven missense mutation functional inference tools on all known pharmacogenetic missense variants contained in the Pharmacogenomics Knowledgebase (PharmGKB) repository. We categorize PharmGKB entries into sub-classes to catalog likely off-target interactions, such that we may compare predictions across different variant annotations.

Results. As previously demonstrated, functional inference tools perform poorly on the complete set of PharmGKB variants, with large numbers of variants incorrectly classified as 'benign'. However, we find substantial differences amongst PharmGKB variant sub-classes, particularly in variants known to cause off-target, type $B$ adverse drug reactions, that are largely unrelated to the main pharmacological action of the drug. Specifically, variants associated with off-target effects (hence referred to as off-target variants) were most often incorrectly classified as 'benign'. These results highlight the importance of understanding the underlying mechanism of pharmacogenetic variants and how variants associated with off-target effects will ultimately require new predictive algorithms.

Conclusion. In this work we demonstrate that functional inference tools perform poorly on pharmacogenetic variants, particularly on subsets enriched for variants causing off-target, type B adverse drug reactions. We describe how to identify variants associated with off-target effects within PharmGKB in order to generate a training set of variants that is needed to develop new algorithms specifically for this class of variant. Development of such tools will lead to more accurate functional predictions and pave the way for the increased wide-spread adoption of pharmacogenetics in clinical practice. 


\section{Efficacy of computational functional effect predictors on 2 idiosyncratic pharmacogenetic variants}

3 Hannah McConnel11 ${ }^{1, \#}$, and T. Daniel Andrews ${ }^{1, \#}$, Matt A. Field ${ }^{2,3}$

$4{ }^{1}$ Genome Informatics Laboratory, Department of Immunology and Infectious Disease, JCSMR, 5 Australian National University, Canberra, ACT, Australia, 0200

$6 \quad{ }^{2}$ Australian Institute of Tropical Health and Medicine and the Centre for Tropical Bioinformatics

7 and Molecular Biology, James Cook University, Smithfield, QLD, Australia, 4878

$8{ }^{3}$ Immunogenomics Lab, Garvan Institute of Medical Research, Victoria Street, Darlinghurst,

9 NSW, Australia, 2010

10 \# Contributed equally to this work

12 Corresponding Authors:

13 T. Daniel Andrews

14 Garran Road, Canberra, ACT, Australia

15 dan.andrews@anu.edu.au

Matt A. Field

14-88 MacGregor Road, Smithfield, Queensland, Australia matt.field@jcu.edu

\section{Abstract}

Background. Pharmacogenetic variation is important to drug responses through diverse and complex mechanisms. Predictions of the functional impact of missense pharmacogenetic variants primarily rely on the degree of sequence conservation between species as a primary discriminator. However, idiosyncratic or off-target drug-variant interactions sometimes involve effects that are peripheral or accessory to the central systems in which a gene functions. Given the importance of sequence conservation to functional prediction tools - these idiosyncratic pharmacogenetic variants may violate the assumptions of predictive software commonly used to infer their effect.

Methods. Here we exhaustively assess the effectiveness of eleven missense mutation functional inference tools on all known pharmacogenetic missense variants contained in the Pharmacogenomics Knowledgebase (PharmGKB) repository. We categorize PharmGKB entries into sub-classes to catalog likely off-target interactions, such that we may compare predictions across different variant annotations.

Results. As previously demonstrated, functional inference tools perform variably across the complete set of PharmGKB variants, with large numbers of variants incorrectly classified as 'benign'. However, we find substantial differences amongst PharmGKB variant sub-classes, 
40 particularly in variants known to cause off-target, type B adverse drug reactions, that are largely

41 unrelated to the main pharmacological action of the drug. Specifically, variants associated with

42 off-target effects (hence referred to as off-target variants) were most often incorrectly classified

43 as 'benign'. These results highlight the importance of understanding the underlying mechanism

44 of pharmacogenetic variants and how variants associated with off-target effects will ultimately

45 require new predictive algorithms.

Conclusion. In this work we demonstrate that functional inference tools perform poorly on pharmacogenetic variants, particularly on subsets enriched for variants causing off-target, type B adverse drug reactions. We describe how to identify variants associated with off-target effects within PharmGKB in order to generate a training set of variants that is needed to develop new algorithms specifically for this class of variant. Development of such tools will lead to more accurate functional predictions and pave the way for the increased wide-spread adoption of pharmacogenetics in clinical practice.

\section{Introduction}

Individual variability in drug response poses a large challenge to safe and effective patient treatment (Meyer 2000; Pirmohamed 2001). Many oncology drugs have be shown to be ineffective in subsets of patients, meaning that often multiple drugs must be tried before an effective treatment is found (Dancey et al. 2012). For example, it is not understood why statins (a class of drugs commonly prescribed for cardiovascular disease) behave differently between individuals (Silva et al. 2006), and can even cause a very severe toxic reaction in a small number of patients (Gabb et al. 2013). It is been estimated that $15-30 \%$ of this variability in drug response is due to genetic factors (Eichelbaum et al. 2006; Pang et al. 2009) however the precise mechanism of such genetic factors is often little understood. Numerous other factors consisting of both genetic and non-genetic components also play a role in variable drug response, including age, ethnicity, gender and differences in alcohol intake.

There are a growing number of databases that aggregate, curate and annotate the increasing body of identified genetic variants that occur in genes that interact with a pharmaceutical (pharmacogenes) (Sim et al. 2011). The Pharmacogenomic Knowledgebase (PharmGKB) (Whirl-Carrillo et al. 2012) is the largest, open database of pharmacogenetic data, and at time of publication, includes information on nearly 150 pathways and over 23,000 individual variant annotations. Variants within PharmGKB are also annotated with effect types (dosage, efficacy, toxicity) and the level of confidence (categories 1-4) of the pharmacogenetic association, with category 1 being the highest. The pharmacogenetic variants included in PharmGKB cover a wide range of mutation types, from nonsynonymous and synonymous single nucleotide variants (SNVs) to non-coding, intergenic and copy number variants. 
80 Predicting the potential functional impact of a missense mutation is necessary, due to the 81 disparity between the number of identified variants and the number that have experimentally82 derived functional data. For missense mutations, this interpretation gap is presently filled by 83 mutation function inference tools, such as PolyPhen2 (Adzhubei et al. 2010), CADD (Kircher et 84 al. 2014) and SIFT (Sim et al. 2012). These function inference prediction tools integrate 85 sequence conservation and, often, structural information to predict whether alterations to the 86 amino-acid sequence are likely to alter the function of a protein (Khan \& Vihinen 2010). These 87 tools are known to suffer from high false positive rates with previous work yielding FPRs of $88>10-65 \%$ for 23 tools that were run across 4880 confirmed ClinVar variants annotated as 89 "pathogenic" or "benign" (Li et al. 2018). Generally, the algorithms work by deriving a multi90 factorial score with higher values (with the exception of SIFT) representative of variants more 91 likely to be damaging to the structure and function of the protein. Several algorithms bin their 92 values into discrete named categories with PolyPhen2 applying labels of 'benign' for scores from 930 and $<0.15$, 'possibly damaging' for scores from 0.15 and $<0.85$ and 'probably damaging' for 94 scores of $0.85-1$. The algorithms are often trained on distinct sets of variants with CADD 95 employing a machine learning model trained on a binary distinction between fixed variants 96 arisen since the human/chimp split and simulated de novo variants. The types of evidence 97 employed by the algorithms are numerous with CADD for example considering 60 annotation 98 sets in total. The annotation sets cover a wide variety of evidence types including sequence 99 conservation (e.g. phastCons, GERP), epigenetic modifications (e.g. DNase-Seq, H3K9Ac), 100 functional prediction (e.g. TF motif, amino acid change), and genetic content (e.g. GC content, 101 CpG content) amongst others. Despite the diversity of evidence types however, sequence 102 conservation remains the most widely used evidence type and to our knowledge all tools 103 incorporate sequence conservation data in their algorithms. Hence, sequence conservation is 104 important and widely used in classifying a variant as either benign or deleterious.

105

106 The reliance of such tools on sequence conservation is critical when considering 107 pharmacogenetic variation. A recent study assessed the effectiveness of eight tools on variants 108 in the RYR1 gene, which is linked to pharmacogenetic disorder malignant hyperthermia (MH) 109 (Schiemann \& Stowell 2016). They compared MH-causative variants and common variants and 110 found none of the prediction programmes could classify all variants correctly as either 111 'damaging' or as 'benign' respectively (84\% - 100\% range for sensitivity and $25 \%$ - $83 \%$ range of 112 specificity). Specific missense mutations have been shown to cause adverse off-target effects 113 with rs1050828 causing glucose-6-phosphate dehydrogenase (G6PD) deficiency which induces 114 haemolytic anaemia from anti-malarial drugs such as primaquine (Gampio Gueye et al. 2019).

115 A broader study appraised mutation functional inference methods across a variety of

116 pharmacogenetic missense variants and also found them to perform poorly with the effect

117 attributed to the ill-suited training sets used to build the models on which the algorithms rely

118 (Zhou et al. 2018b). Such studies led us to examine pharmacogenetic variants in order to

119 identify subclasses that are likely to perform poorly with such tools such as variants associated 120 with adverse drug reactions (ADRs). 
122 ADRs are broadly classified according to general mechanistic distinctions (Patton \& Borshoff

123 2018). Type A reactions are common and their effects are predictable and mostly dose-

124 dependent. Type A reactions relate to interactions of a drug with its primary drug target.

125 Conversely, type B reactions are less common and are mostly unrelated to the main

126 pharmacological action of the drug. Type B reactions, sometimes also called idiosyncratic drug

127 reactions (Uetrecht \& Naisbitt 2013), can be dose-dependent or dose-independent, may be

128 immunologically-mediated and/or may involve off-target drug interactions (Patton \& Borshoff

129 2018). Immune-mediated Type $B$ reactions involve the drug inducing a specific immune

130 response, such as the development of a skin rash commonly caused by administration of

131 penicillin (Weiss \& Adkinson 1988). Off-target drug effects can also occur without an

132 immunological component, such as the interactions of anesthetics with the ryanodine receptor 1

133 (RYR1) protein causing malignant hyperthermia (Robinson et al. 2006).

134

135 We extracted all PharmGKB variants causing missense mutations and assessed the effectiveness

136 of eleven functional inference tools. PharmGKB contains substantial numbers of variants, across

137 all variant evidence levels, that are computationally predicted to be benign. We independently

138 analyzed variant sub-classes associated with Type A and Type B reactions to determine whether

139 the functional inferences differ. We find that most PharmGKB entries incorrectly classified as

140 benign are generally off-target, idiosyncratic variants. As such, using current functional-effect

141 prediction tools may produce poor inferences on idiosyncratic pharmacogenetic variants.

142 Identifying lists of such variants generates a training set suitable to develop and calibrate new

143 algorithms designed for this specific sub-class of variant.

144

\section{Materials \& Methods}

\section{Pharmacogenetic Variant Datasets}

147 A set of pharmacogenetic variants with dbSNP reference cluster identifiers (RS) (Sherry et al.

148 2001) were obtained from PharmGKB (Whirl-Carrillo et al. 2012) and custom overlap code used

149 to combine variant annotations (Field et al. 2015; Waardenberg \& Field 2019). Variants within

150 PharmGKB are classified by gene, type of effect, level of evidence, specific drug, chemical,

151 disease and phenotype.

152

\section{Classification of Off-Target Pharmacogenetic Variants}

154 A simple classification scheme was devised to identify and confirm likely off-target variants

155 (Table 1). Input variants for this classification were the complete set of PharmGKB variants

156 (Whirl-Carrillo et al. 2012). First, of these variants, all synonymous and non-coding variants

157 were excluded, leaving just missense variants. All clinical variants were then filtered for

158 PharmGKB annotations of effect type 'Toxicity/ADR' for any particular chemical and/or drug.

159 Variants were removed if they also had an additional effect type (other than 'Toxicity/ADR') for

160 the same drug. Next, variants were removed if they were present in absorption, distribution, 
161 metabolism, and excretion process genes (ADME; categorized as such in the PharmaADME

162 database; www.pharmaadme.org) or were annotated with Gene Ontology (24) categories of

163 'xenobiotic metabolism process' or with 'transporter'. Lastly, variants not containing entries in

164 ClinVar were removed.

165

166

167

Validation of classification scheme

168

To validate how effective this classification system was at capturing off-target variants, we

169

randomly sampled high-confidence, missense variants from the PharmGKB (Whirl-Carrillo et al.

170 2012) until we derived 30 variants with a known pharmacogenetic mechanism (Supplemental

171

Table S1). For each of these 30 variants we conducted a detailed literature review to manually classify each variant as Type A or Type B. Note this is an additional step not described in Table 1. Manual classification was geared toward being stringent and followed a checklist where the variant was assigned to Type B if it satisfied all of the following criteria: 1) was not a metabolic process gene associated with normal metabolism of the drug, 2) was not in a gene associated with the system the drug is prescribed to target, and 3) did not have a dose effect. These 30 variants were taken as gold-standard data for benchmarking our approach to determine variants associated with off-target effects.

Functional Effect Prediction

For all PharmGKB missense mutations, the predicted functional effect of mutations with SIFT v5.2.2 (Sim et al. 2012), PolyPhen2 v2.2.2 (Adzhubei et al. 2010), CADD v1.5 (Kircher et al. 2014), DANN (pre-calculated results downloaded July 2019) (Quang et al. 2015), FATHMM v2.3 (Shihab et al. 2013), GERP++ v2 (Davydov et al. 2010), MutPred v2 (Li et al. 2009), Mutation Assessor v3 (Reva et al. 2011), Mutation Taster v2 (Schwarz et al. 2014), REVEL v3 (Ioannidis et al. 2016) and PhastCons v1.5 (Siepel et al. 2005) were calculated relative to EnsEMBL canonical transcripts using dbNSFP v4.0a (Liu et al. 2013) and Variant Effect Predictor v94 (VEP) (McLaren et al. 2016) (Supplemental Table S2). Instances where a tool produced no value for a given SNV were recorded as an NA value. ROC curves were obtained for the most-widely used subset of these tools (CADD, PolyPhen2, SIFT, Mutation Assessor, MutPred and REVEL) using the R package ROCR (Sing et al. 2005). All high-confidence PharmGKB variants (classified with the highest level of confidence 'category 1') were input as the positive control while a set of randomly selected common variants (MAF $>0.1$ ) were input as the negative control using the Perl function rand() across the entire set of dbSNP v151 missense variants. The same analysis was next performed using all low confidence PharmGKB variants (classified with the lowest levels of confidence 'category 3' or 'category 4'). Labels were inverted for SIFT due to lower scores representing likely damaging mutations and CADD and Mutation Assessor scores were scaled into the range of 0-1. Area under the curve and the Matthews Correlation Coefficient were calculated using the R-package 
201 ROCR performance function. Code used in all analyses are available at

202 https://github.com/mattmattmattmatt/pharmaco_paper.

203

204

\section{Results}

205

\section{Distributions of pharmacogenetic variant functional inferences}

206

Functional inference scores were obtained for 561 missense single nucleotide variants (SNVs)

207 present in PharmGKB, that also had dbSNP cluster identifiers. Predictions were made for each SNV with eleven different prediction tools (SIFT, PolyPhen2, CADD, DANN, FATHMM, GERP++, MutPred, Mutation Assessor, Mutation Taster, REVEL and PhastCons) (Supplemental Table S2). The distributions of scores from the most widely-used subset of these tools (CADD, PolyPhen2, SIFT, Mutation Assessor, Mutation Predictor and REVEL) are plotted with variants grouped by major PharmGKB category 1-4 (Figure 1). The predictions calculated for these functional variants ranged widely from benign to deleterious. While four of the tools generate scores in the range of $0-1$ (with 1 most damaging expect SIFT 0 most damaging). Mutation Assessor and CADD employ a range of positive values with CADD calculating a Phred-quality score. For example, a CADD score of 20 implies the variant is ranked in the top $0.1 \%$ of all possible variant scores based on all possible changes in the human genome (CADD score of 10 is top $1 \%$, CADD score of 20 is top $0.1 \%$, CADD score of 30 is top $0.01 \%$, etc.). For comparison to expected background levels, we also selected a random set of 2155 common human missense SNVs with assigned RS cluster identifiers. Overall, the distribution of random variants mirrors our previous work with Polyphen2 exhibiting a characteristic hourglass curve with very few intermediate values (Andrews et al. 2012). These tools represent a broad range of methodologies available for mutation functional prediction and the categories of information used by each tool are annotated in Figure 1 as seq (sequence conservation), struct (protein structural metrics), and ens (ensemble tool that integrates individual tools). The results demonstrate how some of the highest confidence PharmGKB variants annotated as functionally important are predicted to be benign. Of the 119 highest confidence category 1 variants, the majority are predicted to be deleterious by PolyPhen2 (median score 0.996), however 5 variants were classified benign (rs116855232, rs1057910, rs121909041, rs3745274, and rs1050828). The 183 variants in category 2 had a much broader range of predicted functional effects with 33 variants predicted as benign and an overall median score of 0.138 , even less than the median score of 0.245 for the randomly selected variants. Similarly, the distribution of functional effect predictions in category 3 was strongly skewed towards benign variants (PolyPhen2 median score 0.012 ) and category 4 had a distribution very similar to the random variant set (PolyPhen2 median score 0.319). Category 1 variants were compared to category 2, 3 and 4 variants using a Mann-Whitney $U$ test yielding $p$ values of $5.55 \mathrm{e}-07,2.19 \mathrm{e}-24$, and $1.03 \mathrm{e}-18$ respectively.

237 examine whether these mutations act on the core protein function. rs116855232 is found to cause 
241

242

243

244

245

246

247

248

249

250

251

252

253

254

255

256

257

258

259

260

261

262

263

264

265

266

267

268

269

270

271

272

273

274

275

276

277

278

279

280

diphosphatase activity affecting the metabolism of Thiopurines. rs121909041 causes a missense mutation in CFTR and is associated with increased response to ivacaftor for the treatment of cystic fibrosis. rs 1050828 causes a Va198Met mutation in G6PD, a gene which helps protect cells from oxidative damage. This mutation causes an adverse off-target reaction with the G6PD deficiency inducing haemolytic anaemia from anti-malarial drugs such as primaquine. The remaining two variants occur in the cytochrome p450 CYP2 family of enzymes which are important in a variety of physiological and toxicological processes. rs 1057910 causes a Ile359Leu change in CYP2C9 and results in poor metabolism for a wide variety of drugs used in the treatment of diabetes, epilepsy, and cardiovascular disease. rs3745274 causes a Gln172His change in CYP2C6 and reduces response to various HIV infection treatments. These examples illustrate how in some cases the variants do not act on core protein function.

To better assess the performance of the individual algorithms on high confidence (category 1) pharmacogenetic variants, ROC plots were generated for CADD, PolyPhen2, SIFT, Mutation Assessor, Mutation Predictor and REVEL (Figure 2). Area under the curve (AUC) and Matthews Correlation Coefficient were calculated (Table 2). Overall, Mutation Predictor had the highest AUC at 0.975 followed by REVEL at 0.942 , PolyPhen2 at 0.852 and the remaining algorithms raging from 0.728-0.774. REVEL had the highest Matthews Correlation Coefficient at 0.794, followed by Mutation Predictor at 0.788 and the remaining algorithms raging from 0.321-0.489. We next performed the identical analysis on low confidence (category 3 and 4) variants and uniformly found lower AUCs ranging from 0.397 to 0.682. Overall, the AUCs are significantly different ( $p$-value 0.00512 ) between the high-confidence and low-confidence variants.

In addition to missense mutations, tools such as CADD are able to generate scores for other variant types such as non-coding SNVs. While detailed analysis of this type of variant is beyond the scope of this study, we identified 14 PharmGKB high-quality category 1 and 2 non-coding variants and generated CADD scores. The median CADD score was 14.0, well below the average of 27.2 for category 1 PharmGKB and even less than the 22.1 for the random $2155 \mathrm{dbSNP}$ variants.

\section{Classification of variation in pharmacogenes to detect off-target effects} A prior study (Zhou et al. 2018b) demonstrated that functional prediction tools do not perform well across all pharmacogenetic variation. We hypothesized however, that the performance of the tools would differ across subtypes of pharmacogenetic variation, and that Type B pharmacogenetic variants associated with off-target effects in particular would often be predicted to be benign. To investigate this possibility, we devised a simple classification system to enrich for Type B variants from PharmGKB (described in Materials and Methods and summarized in Table 1). Our starting data was all possible PharmGKB variants found to cause missense mutations. In order to discern how effective this classification system was at capturing Type B variants, we randomly sampled from the starting set of PharmGKB variants until we derived 30 variants classified as category 1 or 2 from distinct genes with a published pharmacogenetic 
281

282

283

284

285

286

287

288

289

290

291

292

293

294

295

296

297

298

299

300

301

302

303

304

305

306

307

308

309

310

311

312

313

314

315

316

317

318

319

320

mechanism (Supplemental Table S1). While not exhaustive, this manually-intensive process covered almost half of the 61 total genes in pharmGKB containing variants with evidence levels of either category 1 or 2 . For each selected variant we performed a detailed literature review in order to determine whether each variant should be classified as Type A or Type B. Of the 30 variants, nine were determined to be Type B variants (30\%). We next applied our simplified classification scheme (Table 1) to the same 30 variants to identify candidate Type B variants (Supplementary Table S1) which identified eight putative variants. Of these eight, five were true positive Type B variants (with no false negatives) and three were false positives. Given this retention of Type B variants from the unfiltered variant pool $(9 / 30=0.3)$ to the enriched pool $(5 / 8=0.63)$, we estimate from this data that this classification scheme yields a 2.1 -fold $(0.63 / 0.3)$ enrichment of Type B missense variants, with a sensitivity of $63 \%(5 / 8)$ and a specificity of $100 \%(21 / 21)$. Subsequently, using our simplified classification scheme, we analysed the full set of 561 PharmGKB variants causing missense mutations yielding 142 candidate Type B variants (Supplemental Table S3).

\section{Discussion}

In this work we have appraised whether pharmacogenetic variants associated with Type B, offtarget effects are consistently predicted to be less deleterious than other functionally-important variants. We find this to be the case and postulate that this results arises from the reliance of the current generation of missense mutation inference tools on sequence conservation information. Generally, when a deeply conserved genetic element is found to be mutated, this will result in this mutation being predicted to be deleterious. However, should a nucleotide not be conserved across deep evolutionary distances, mutations of that nucleotide are likely to be predicted to be 'benign'. Should that nucleotide code for an amino acid or otherwise directly interact with a drug which confers a life-saving benefit, however, this would clearly not be the case.

We further devised a simple classification scheme to divide pharmacogenetic variants into Type A or Type B. Classifying pharmacogenetic variants is important as unless a pharmacogenetic variant is related to the evolved functions of a gene, then no information is present in the ancestral sequence record to inform on predicted functional importance. Many genes that interact with drugs (pharmacogenes) contain variants which generate Type A ADRs. For these

Type A pharmacovariants, the drug is just a xenobiotic compound which the target-protein acts upon. Variants which adversely affect the function of the pharmacogene should be correctly classified as 'deleterious' by the current generation of functional inference tools. This is supported by previous work showing an association between the residual evidence intolerance score (RVIS which measure the tolerance of a gene to mutations) and targets of approved drugs (Nelson et al. 2015), however it is unknown whether this holds for off-target variants. In our classification, we define Type B variants as those that have only Toxicity/ADR effects, and appear in genes not annotated as part of an ADME process, GO term "xenobiotic metabolic process" GO term "transporter", but do have a known mechanism which has been implicated 
321 with an idiosyncratic drug/protein interaction. Variants that cause a type B or off-target effect are

322 much less likely to be subject to the same selection pressures as those of type A, meaning they

323 may be incorrectly classified as 'benign' due to the lack of observed sequence conservation.

324 Indeed, we show most off-target pharmacogenetic variants of this type are predicted to be

325 functionally unimportant and will be missed using current tools. Zhou et al (Zhou et al. 2018a)

326 similarly observed that the genes containing many pharmacogenetic variants are often poorly

327 conserved, making the reliance of the algorithms on sequence conservation alone problematic.

328 The quality of the multiple sequence alignments is also important with the class of multiple

329 sequence alignment algorithm selected shown to substantially impact downstream analyses

330 (Blackburne \& Whelan 2013). An additional consideration is the constraints imposed by domain

331 structure on missense mutations across the human genome (MacGowan et al. 2017). MacGowan

332 et al. identified regions of the genome depleted of missense mutations and while most such

333 regions were conserved across species, they identified regions that are not conserved yet were

334 enriched for pathogenic variant, ligands, and DNA and protein binding interactions. Such

335 variants cannot be accurately predicted using tools that rely solely on sequence conservation and

336 require additional information such as genome-wide missense depletion scores. Similarly

337 pharmacogenetic variants that are not conserved across species will require additional

338 information for existing tools or even new tools altogether to accurately predict their functional

339 impact. Given the importance of pharmacogenetic variation and the numerous nature of Type B

340 pharmacovariants, new methods are urgently needed to capture this important class of variation.

341

342 While sequence conservation is a useful metric for predicting the impact of many variants, we

343 have shown for certain subclasses of variants it is not suitable. However, without using sequence

344 conservation information as a primary discriminator, what methods and datasets are available to

345 differentiate between truly benign and functionally important variation causing off-target effects?

346 In hopes of finding new ways to predict damaging missense mutations, researchers are

347 increasingly applying machine learning techniques to improve functional prediction algorithms

348 particularly for identifying disease causing variants (Kalinin et al. 2018). However, for

349 pharmacogenetic variants options are limited. A recent study reported improved sensitivity and

350 specificity using a functionality prediction framework optimized for pharmacogenetic variants

351 however no code has yet been released to independently assess this claim (Zhou et al. 2018b).

352 Regardless of the eventual outcome, the ability to accurately predict pharmacogenetic variants

353 associated with off-target effects is critical for the increased adoption of pharmacogenetics in

354 clinical practice.

355

356 Conclusions

357 Pharmacogenetic missense variants represent a complex set of genetic factors with highly diverse

358 functional mechanisms that influence drug efficacy. Here we assessed the performance of

359 functional prediction tools (all which rely to some degree on sequence conservation over deep

360 evolutionary timescales) on different subsets of pharmacogenetic variants. Our analysis 
361 confirms that variant sets enriched for off-target, type B adverse drug reactions perform

362 significantly worse than both randomly selected common variants (MAF $>0.1)$ and variants

363 enriched for Type A reactions that relate to interactions of a drug with its' primary drug target.

364 We describe a simple method to identify candidate variants associated with type B reactions and

365 note that the majority are predicted to be benign and functionally unimportant. Generating a

366 subset of such variants will enable the development of urgently needed new methods that can

367 accurately detect and predict the impact of all pharmacogenetic variation.

368

369

\section{Acknowledgements}

370

We thank the National Computational Infrastructure (Australia) for continued access to

371

significant computation resources and technical expertise.

372

373

374

375

376

References

377

378

379

380

381

382

383

384

385

386

387

388

389

390

391

392

393

394

395

396

397

398

399

400

401

402

403

404

405

406

Adzhubei IA, Schmidt S, Peshkin L, Ramensky VE, Gerasimova A, Bork P, Kondrashov AS, and Sunyaev SR. 2010. A method and server for predicting damaging missense mutations. Nat Methods 7:248-249.

Andrews TD, Whittle B, Field MA, Balakishnan B, Zhang Y, Shao Y, Cho V, Kirk M, Singh M, Xia Y, Hager J, Winslade S, Sjollema G, Beutler B, Enders A, and Goodnow CC. 2012. Massively parallel sequencing of the mouse exome to accurately identify rare, induced mutations: an immediate source for thousands of new mouse models. Open Biol 2:120061.

Blackburne BP, and Whelan S. 2013. Class of multiple sequence alignment algorithm affects genomic analysis. Mol Biol Evol 30:642-653.

Dancey JE, Bedard PL, Onetto N, and Hudson TJ. 2012. The genetic basis for cancer treatment decisions. Cell 148:409-420.

Davydov EV, Goode DL, Sirota M, Cooper GM, Sidow A, and Batzoglou S. 2010. Identifying a high fraction of the human genome to be under selective constraint using GERP++. PLoS Comput Biol 6:e1001025.

Eichelbaum M, Ingelman-Sundberg M, and Evans WE. 2006. Pharmacogenomics and individualized drug therapy. Annu Rev Med 57:119-137.

Field MA, Cho V, Andrews TD, and Goodnow CC. 2015. Reliably Detecting Clinically Important Variants Requires Both Combined Variant Calls and Optimized Filtering Strategies. PLoS One 10:e0143199.

Gabb GM, Vitry A, Condon J, Limaye V, and Alhami G. 2013. Serious statin-associated myotoxicity and rhabdomyolysis in Aboriginal and Torres Strait Islanders: a case series. Intern Med J 43:987-992.

Gampio Gueye NS, Peko SM, Nderu D, Koukouikila-Koussounda F, Vouvoungui C, Kobawila SC, Velavan TP, and Ntoumi F. 2019. An update on glucose-6-phosphate dehydrogenase deficiency in children from Brazzaville, Republic of Congo. Malar $\mathrm{J}$ 18:57.

loannidis NM, Rothstein JH, Pejaver V, Middha S, McDonnell SK, Baheti S, Musolf A, Li Q, Holzinger E, Karyadi D, Cannon-Albright LA, Teerlink CC, Stanford JL, Isaacs WB, Xu J, 
407

408

409

410

411

412

413

414

415

416

417

418

419

420

421

422

423

424

425

426

427

428

429

430

431

432

433

434

435

436

437

438

439

440

441

442

443

444

445

446

447

448

449

450

451

452

453

454

455
Cooney KA, Lange EM, Schleutker J, Carpten JD, Powell IJ, Cussenot O, Cancel-Tassin G, Giles GG, Maclnnis RJ, Maier C, Hsieh CL, Wiklund F, Catalona WJ, Foulkes WD, Mandal D, Eeles RA, Kote-Jarai Z, Bustamante CD, Schaid DJ, Hastie T, Ostrander EA, Bailey-Wilson JE, Radivojac P, Thibodeau SN, Whittemore AS, and Sieh W. 2016. REVEL: An Ensemble Method for Predicting the Pathogenicity of Rare Missense Variants. Am J Hum Genet 99:877-885.

Kalinin AA, Higgins GA, Reamaroon N, Soroushmehr S, Allyn-Feuer A, Dinov ID, Najarian K, and Athey BD. 2018. Deep learning in pharmacogenomics: from gene regulation to patient stratification. Pharmacogenomics 19:629-650.

Khan S, and Vihinen M. 2010. Performance of protein stability predictors. Hum Mutat 31:675684.

Kircher M, Witten DM, Jain P, O'Roak BJ, Cooper GM, and Shendure J. 2014. A general framework for estimating the relative pathogenicity of human genetic variants. Nat Genet 46:310-315.

Li B, Krishnan VG, Mort ME, Xin F, Kamati KK, Cooper DN, Mooney SD, and Radivojac P. 2009. Automated inference of molecular mechanisms of disease from amino acid substitutions. Bioinformatics 25:2744-2750.

Li J, Zhao T, Zhang Y, Zhang K, Shi L, Chen Y, Wang X, and Sun Z. 2018. Performance evaluation of pathogenicity-computation methods for missense variants. Nucleic Acids Res 46:7793-7804.

Liu X, Jian X, and Boerwinkle E. 2013. dbNSFP v2.0: a database of human non-synonymous SNVs and their functional predictions and annotations. Hum Mutat 34:E2393-2402.

MacGowan SA, Madeira F, Britto-Borges T, Schmittner MS, Cole C, and Barton GJ. 2017. Human Missense Variation is Constrained by Domain Structure and Highlights Functional and Pathogenic Residues. bioRxiv:127050.

McLaren W, Gil L, Hunt SE, Riat HS, Ritchie GR, Thormann A, Flicek P, and Cunningham F. 2016. The Ensembl Variant Effect Predictor. Genome Biol 17:122.

Meyer UA. 2000. Pharmacogenetics and adverse drug reactions. Lancet 356:1667-1671.

Nelson MR, Tipney H, Painter JL, Shen J, Nicoletti P, Shen Y, Floratos A, Sham PC, Li MJ, Wang J, Cardon LR, Whittaker JC, and Sanseau P. 2015. The support of human genetic evidence for approved drug indications. Nat Genet 47:856-860.

Pang GS, Wang J, Wang Z, and Lee CG. 2009. Predicting potentially functional SNPs in drugresponse genes. Pharmacogenomics 10:639-653.

Patton K, and Borshoff DC. 2018. Adverse drug reactions. Anaesthesia 73 Suppl 1:76-84.

Pirmohamed M. 2001. Pharmacogenetics and pharmacogenomics. Br J Clin Pharmacol 52:345347.

Quang D, Chen Y, and Xie X. 2015. DANN: a deep learning approach for annotating the pathogenicity of genetic variants. Bioinformatics 31:761-763.

Reva B, Antipin Y, and Sander C. 2011. Predicting the functional impact of protein mutations: application to cancer genomics. Nucleic Acids Res 39:e118.

Robinson R, Carpenter D, Shaw MA, Halsall J, and Hopkins P. 2006. Mutations in RYR1 in malignant hyperthermia and central core disease. Hum Mutat 27:977-989.

Schiemann AH, and Stowell KM. 2016. Comparison of pathogenicity prediction tools on missense variants in RYR1 and CACNA1S associated with malignant hyperthermia. $\mathrm{Br} \mathrm{J}$ Anaesth 117:124-128.

Schwarz JM, Cooper DN, Schuelke M, and Seelow D. 2014. MutationTaster2: mutation prediction for the deep-sequencing age. Nat Methods 11:361-362.

Sherry ST, Ward MH, Kholodov M, Baker J, Phan L, Smigielski EM, and Sirotkin K. 2001. dbSNP: the NCBI database of genetic variation. Nucleic Acids Res 29:308-311.

PeerJ reviewing PDF | (2020:07:50571:3:0:NEW 11 May 2021) 
456

457

458

459

460

461

462

463

464

465

466

467

468

469

470

471

472

473

474

475

476

477

478

479

480

481

482

483

484

485

486

487

488

489

490

491

492

493

494

495

496

497

498

499

500

501

502

503

504

Shihab HA, Gough J, Cooper DN, Stenson PD, Barker GL, Edwards KJ, Day IN, and Gaunt TR. 2013. Predicting the functional, molecular, and phenotypic consequences of amino acid substitutions using hidden Markov models. Hum Mutat 34:57-65.

Siepel A, Bejerano G, Pedersen JS, Hinrichs AS, Hou M, Rosenbloom K, Clawson H, Spieth J, Hillier LW, Richards S, Weinstock GM, Wilson RK, Gibbs RA, Kent WJ, Miller W, and Haussler D. 2005. Evolutionarily conserved elements in vertebrate, insect, worm, and yeast genomes. Genome Res 15:1034-1050.

Silva MA, Swanson AC, Gandhi PJ, and Tataronis GR. 2006. Statin-related adverse events: a meta-analysis. Clin Ther 28:26-35.

Sim NL, Kumar P, Hu J, Henikoff S, Schneider G, and Ng PC. 2012. SIFT web server: predicting effects of amino acid substitutions on proteins. Nucleic Acids Res 40:W452457.

Sim SC, Altman RB, and Ingelman-Sundberg M. 2011. Databases in the area of pharmacogenetics. Hum Mutat 32:526-531.

Sing T, Sander O, Beerenwinkel N, and Lengauer T. 2005. ROCR: visualizing classifier performance in R. Bioinformatics 21:3940-3941.

Uetrecht J, and Naisbitt DJ. 2013. Idiosyncratic adverse drug reactions: current concepts. Pharmacol Rev 65:779-808.

Waardenberg AJ, and Field MA. 2019. consensusDE: an R package for assessing consensus of multiple RNA-seq algorithms with RUV correction. PeerJ 7:e8206.

Weiss ME, and Adkinson NF. 1988. Immediate hypersensitivity reactions to penicillin and related antibiotics. Clin Allergy 18:515-540.

Whirl-Carrillo M, McDonagh EM, Hebert JM, Gong L, Sangkuhl K, Thorn CF, Altman RB, and Klein TE. 2012. Pharmacogenomics knowledge for personalized medicine. Clin Pharmacol Ther 92:414-417.

Zhou Y, Fujikura K, Mkrtchian S, and Lauschke VM. 2018a. Computational Methods for the Pharmacogenetic Interpretation of Next Generation Sequencing Data. Front Pharmacol 9:1437.

Zhou Y, Mkrtchian S, Kumondai M, Hiratsuka M, and Lauschke VM. 2018b. An optimized prediction framework to assess the functional impact of pharmacogenetic variants. Pharmacogenomics $\mathrm{J}$.

Figure 1 - Distribution of functional effect scores of PharmGKB variants predicted by six mutation effect inference tools. Boxplots shown are of a) CADD Phred score, b) PolyPhen2 score, c) SIFT score, d) Mutation Assessor score, e) MutPred score and f) REVEL score. Scores are plotted for each tool in variant confidence categories (from 1 (highest) to 4 (lowest)) assigned by the PharmGKB annotation. Each tool is annotated with the information types it employs to make predictions - Seq: sequence conservation, Struct: protein structural metrics, Ens: an ensemble tool that integrates results of several individual tools. Each tool employs categorical cutoffs with the most damaging category colored as red.

Figure 2 - Receiver operator curves (ROC) for CADD, Mutation Assessor, MutPred, PolyPhen2, REVEL, and SIFT generated with ROCR. All high-confident PharmGKB Category 1 missense variants (Type A enriched) were input as the positive set while a set of randomly selected common variants (MAF > 0.1) were input as the negative set using the Perl function rand() across the entire set of dbSNP missense variants. The same analysis was also performed for all low-confidence PharmGKB Category 3 or 4 missense variants (Type B enriched). Labels were inverted for SIFT due to lower scores representing likely damaging mutations and CADD and Mutation Assessor scores were scaled into the range of 0-1.

Peer) reviewing PDF | (2020:07:50571:3:0:NEW 11 May 2021) 
505

506

507 


\section{Figure 1}

Functional inference scores across all PharmGKB variants grouped by confidence level.

Distribution of functional effect scores of PharmGKB variants predicted by six mutation effect inference tools. Boxplots shown are of a) CADD Phred score, b) PolyPhen2 score, c) SIFT score, d) Mutation Assessor score, e) MutPred score and f) REVEL score. Scores are plotted for each tool in variant confidence categories (from 1 (highest) to 4 (lowest)) assigned by the PharmGKB annotation. Each tool is annotated with the information types it employs to make predictions - Seq: sequence conservation, Struct: protein structural metrics, Ens: an ensemble tool that integrates results of several individual tools. Each tool employs categorical cutoffs with the most damaging category colored as red.
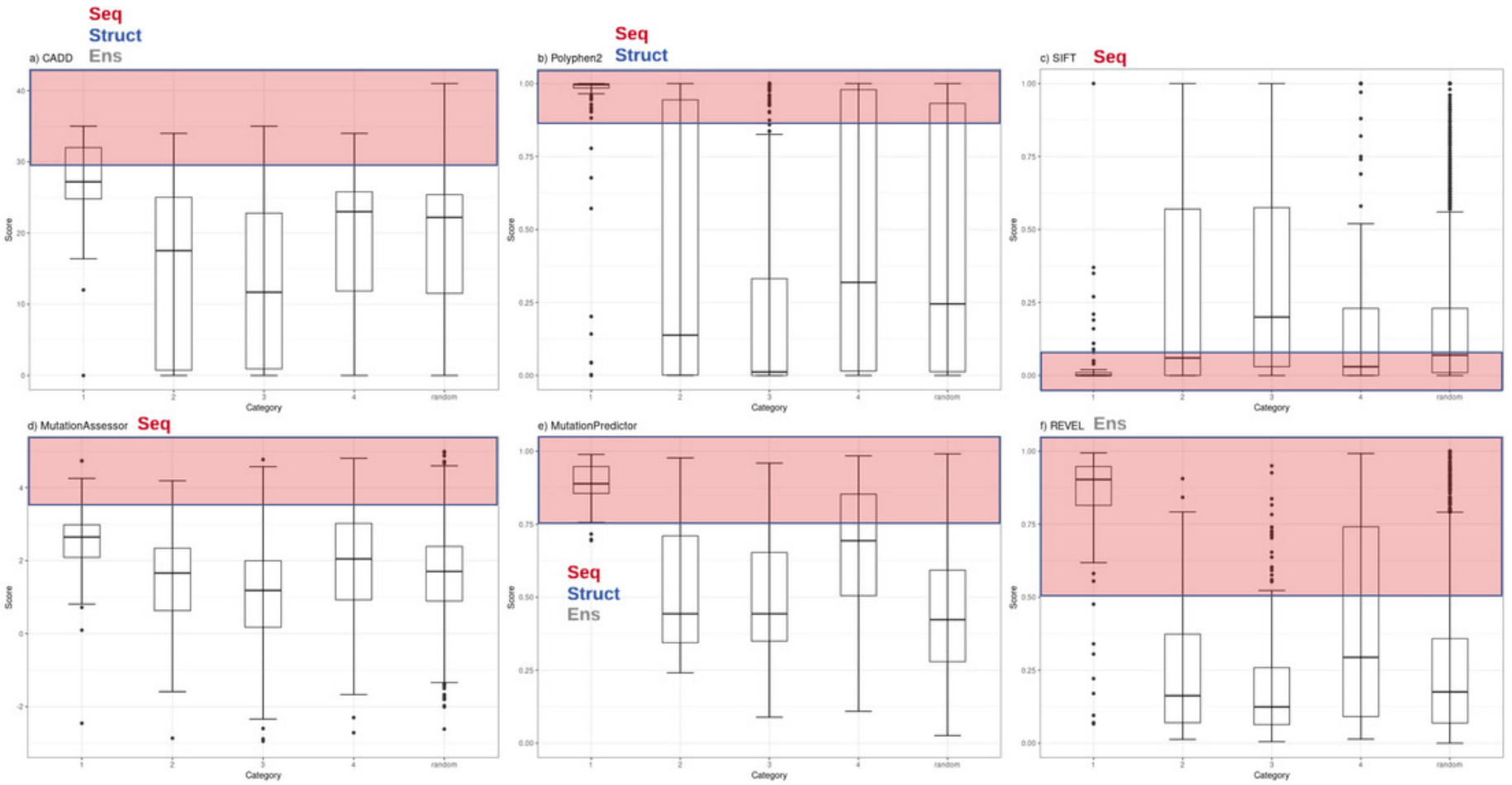


\section{Figure 2}

Receiver operator curves (ROC) for six functional inference tools vs Type A and Type B enriched pharmacogenetic variants.

Receiver operator curves (ROC) for CADD, Mutation Assessor, MutPred, PolyPhen2, REVEL, and SIFT generated with ROCR. All high-confident PharmGKB Category 1 missense variants (Type $A$ enriched) were input as the positive set while a set of randomly selected common

variants (MAF > 0.1) were input as the negative set using the Perl function rand() across the entire set of dbSNP missense variants. The same analysis was also performed for all lowconfidence PharmGKB Category 3 or 4 missense variants (Type B enriched). Labels were inverted for SIFT due to lower scores representing likely damaging mutations and CADD and Mutation Assessor scores were scaled into the range of 0-1. 

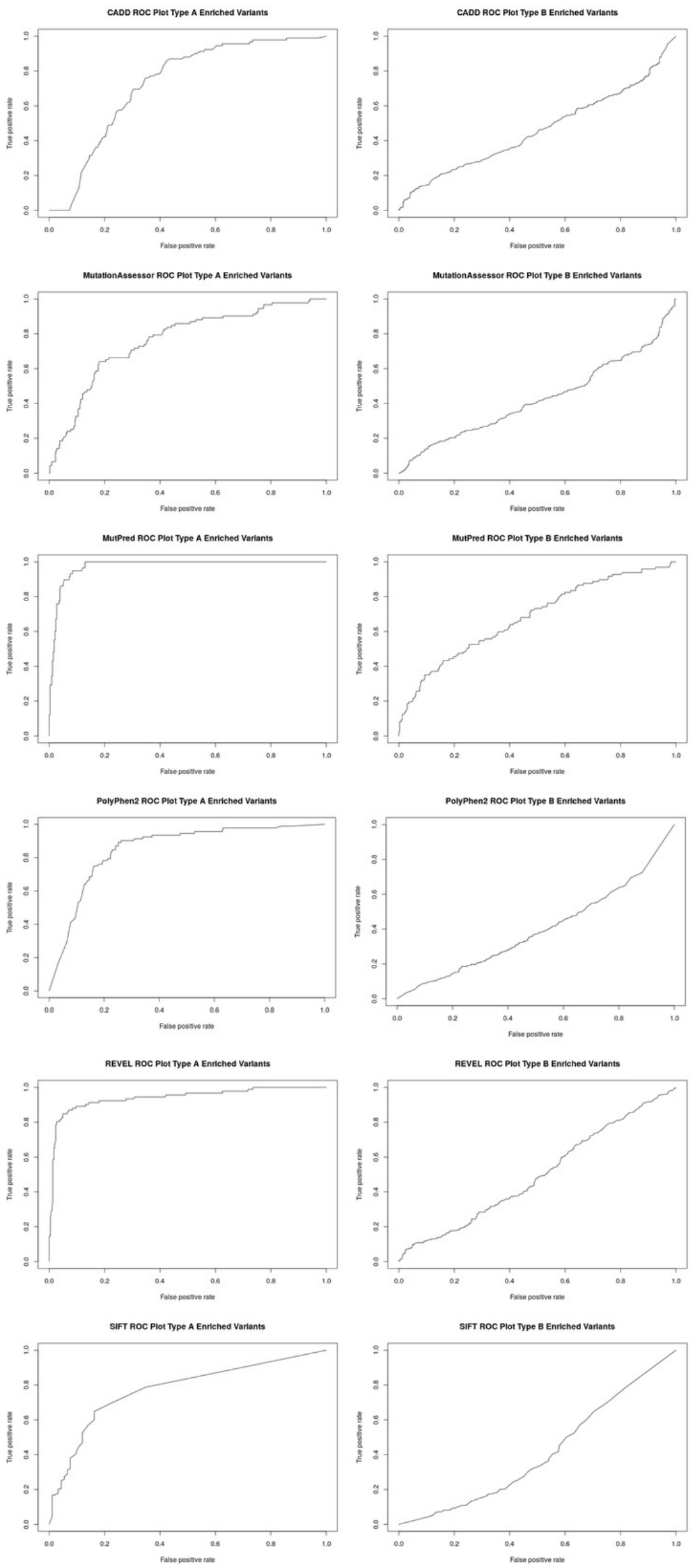

Peer) reviewing PDF | (2020:07:50571:3:0:NEW 11 May 2021) 


\section{Table 1 (on next page)}

Classification criteria used to identify off-target pharmacogenetic variants from the PharmGKB database. 


\begin{tabular}{|c|c|c|}
\hline Step & Filter & Number Variants \\
\hline 1 & $\begin{array}{l}\text { Exclude synonymous and } \\
\text { non-coding variants }\end{array}$ & 561 \\
\hline 2 & $\begin{array}{l}\text { Include variants that have } \\
\text { type:toxicity/ADR }\end{array}$ & 339 \\
\hline 3 & $\begin{array}{l}\text { For drug and gene pairs, } \\
\text { exclude variants with } \\
\text { additional effect types other } \\
\text { than Toxicity/ADR }\end{array}$ & 273 \\
\hline 4 & $\begin{array}{l}\text { Gene containing variant is } \\
\text { NOT an ADME process gene } \\
\text { OR annotated in GO with } \\
\text { 'xenobiotic metabolic } \\
\text { process' OR 'transporter' }\end{array}$ & 196 \\
\hline 5* & $\begin{array}{l}\text { Variant not containing an } \\
\text { entry in clinVAR }\end{array}$ & 142 \\
\hline
\end{tabular}

1

2 *For 30 variants used as a truth set, we performed an additional detailed literature search to

3 definitively classify each variant as type A or type B. 


\section{Table 2 (on next page)}

AUC and MCC from functional inference tools for PharmGKB category 1 (Type $A$ enriched) vs PharmGKB category 3 and 4 (Type B enriched) pharmacogenetic variants. 


\begin{tabular}{|l|l|l|l|}
\hline Algorithm & $\begin{array}{l}\text { Type A or Type B } \\
\text { Enriched }\end{array}$ & $\begin{array}{l}\text { Area Under Curve } \\
\text { (AUC) }\end{array}$ & $\begin{array}{l}\text { Matthews Correlation } \\
\text { Coefficient (MCC) }\end{array}$ \\
\hline PolyPhen2 & Type A & 0.852 & 0.489 \\
\hline MutPred & Type A & 0.975 & 0.788 \\
\hline REVEL & Type A & 0.942 & 0.794 \\
\hline SIFT & Type A & 0.774 & 0.358 \\
\hline CADD & Type A & 0.728 & 0.321 \\
\hline Mutation Assessor & Type A & 0.763 & 0.396 \\
\hline PolyPhen2 & Type B & 0.397 & 0.00338 \\
\hline MutPred & Type B & 0.682 & 0.300 \\
\hline REVEL & Type B & 0.498 & 0.106 \\
\hline SIFT & Type B & 0.410 & -0.0400 \\
\hline CADD & Type B & 0.461 & 0.118 \\
\hline Mutation Assessor & Type B & 0.427 & 0.0764 \\
\hline Average Type A & & $\mathbf{0 . 8 3 9}$ & $\mathbf{0 . 5 2 4}$ \\
\hline Average Type B & & $\mathbf{0 . 4 7 9}$ & $\mathbf{0 . 0 9 4}$ \\
\hline
\end{tabular}

